\title{
Characteristics of Three Person-to-Person Transmission Clusters of Severe Fever with Thrombocytopenia Syndrome in Southeastern China
}

\author{
Mingyong Tao, ${ }^{1} \dagger$ Ying Liu, ${ }^{2}$ † Feng Ling, ${ }^{2}$ † Rong Zhang, ${ }^{2}$ Xuguang Shi, ${ }^{2}$ Jiangping Ren, ${ }^{2}$ Song Guo, ${ }^{2}$ Jimin Sun,${ }^{2 \star}$ and \\ Jianmin Jiang ${ }^{2 \star}$ \\ ${ }^{1}$ Ningbo University, Ningbo, China; ${ }^{2}$ Key Laboratory of Vaccine, Prevention and Control of Infectious Disease of Zhejiang Province, Zhejiang \\ Provincial Center for Disease Control and Prevention, Hangzhou, China
}

\begin{abstract}
Severe fever with thrombocytopenia syndrome (SFTS) is an emerging infectious disease worldwide. It can be transmitted from person to person, and the fatality rate is very high. During this study, three SFTS clusters including 12 associated cases were identified in three counties in Zhejiang Province from 2018 to 2020. The median age of the three index patients was 70 years, and that of secondary case patients was 59 years. Of note, the mortality rate of the index patients was $100 \%$. The mortality rate of secondary case patients was $11 \%$. The total secondary attack rate (SAR) was $30 \%$ (9/30). The SARs of cluster A, cluster B, and cluster C were $38 \%(3 / 8), 21 \%(3 / 14)$, and 38\% (3/8), respectively. Additionally, the interval from onset to diagnosis was 4 days. The intervals from disease onset to confirmation of the index cases and secondary cases were 7 days and 4 days, respectively. All secondary case patients had a history of close contact with blood or body fluids of the index patients. These results indicate that SFTS patients should not be discharged until recovery. When SFTS patients die, the corpses should be transferred directly from the hospital to the crematorium for cremation by persons wearing proper protective equipment to prevent virus transmission.
\end{abstract}

\section{INTRODUCTION}

Severe fever with thrombocytopenia syndrome (SFTS) is an emerging infectious disease that was first reported in Hubei Province and Henan Province in China in 2009. The disease is caused by SFTS virus (SFTSV), which belongs to genus Phlebovirus, family Phenuiviridae, order Bunyavirales. ${ }^{1}$ In 2017, the WHO listed SFTSV as one of the nine infectious diseases on the priority list because the SFTSV poses an enormous threat to human health. The major clinical manifestations of SFTS infection include acute onset of fever, thrombocytopenia, leukocytopenia, gastrointestinal symptoms, gingival hemorrhage, conjunctival congestion, and central nervous system manifestations. ${ }^{2-4}$ Most patients with SFTS cases recover after a self-limiting clinical course; only a small proportion of patients die of diffuse intravascular coagulation (DIC) and multiple organ failure. ${ }^{5,6}$ SFTSV and SFTS cases have been identified and subsequently reported in South Korea, Japan, and Vietnam. ${ }^{7-9}$ Cases similar to SFTS infection have been reported in the United States, but the agent for these cases was alleged to be Heartland virus. ${ }^{10}$ Moreover, a novel Zwiesel bat banyangvirus that is closely related to SFTSV has been detected in Germany. ${ }^{11}$

Arthropods, especially ticks, are regarded as the reservoir for SFTSV. SFTSV RNA was detectable in Haemaphysalis longicornis, Rhipicephalus microplus, Amblyomma testudinarium, and Ixodes nipponensis. ${ }^{12,13}$ The search for SFTSV animal reservoirs is ongoing, and the virus has been isolated from a wide range of animals, including cattle, goats, dogs, pigs, and chickens. ${ }^{14-16}$ Of interest, one study reported that a case of SFTS was directly caused by an infected cat. ${ }^{17}$

Some SFTS clusters have occurred in China, and direct person-to-person contact with blood or body fluids has been considered the transmission route. ${ }^{18-21}$ Previous studies

\footnotetext{
* Address correspondence to Jimin Sun or Jianmin Jiang, 3399 Binsheng Road, Binjiang District, Hangzhou, China. E-mails: jmsun@cdc.zj.cn or jmjiang@cdc.zj.cn

†These authors contributed equally to this work.
}

have reported that the virus could be transmitted through aerosols. ${ }^{22,23}$

The majority of SFTS cases have been observed in East Asia. More than 7,000 laboratory-confirmed SFTS cases and 355 deaths have been reported in China, and the annual confirmed case number is increasing. ${ }^{24}$ Zhejiang Province, located in southeastern China, has reported more than 500 SFTS cases from 2011 to 2020 . Notably, some SFTS clusters occurred in Zhejiang Province. This is of great public health significance in Zhejiang. During April and May 2014, three sisters contracted SFTSV and died. In May 2014, a total of 13 SFTS patients acquired infection through contact with the blood or bloody secretions of the index patient. ${ }^{22,25}$ During this study, we examined the three latest SFTS clusters in Zhejiang Province and summarized their epidemiological characteristics to help prevent SFTS outbreaks.

\section{MATERIALS AND METHODS}

Case definition. According to the guidelines for SFTS prevention and control issued by the National Health Commission of the People's Republic of China in 2010, SFTS is suspected when patients have acute onset of fever $\left(\geq 38.0^{\circ} \mathrm{C}\right)$, symptoms such as diarrhea, confusion, nausea, and bleeding, epidemiological risk factors (farmer or exposed to ticks 2 weeks before illness onset), thrombocytopenia, and leukocytopenia. In addition to laboratory confirmation, a suspected SFTS case should fulfill at least one of the following criteria: positive nucleic acid test results; positive seroconversion of IgG or four-fold increase in the antibody titers between the acute phase and recovery phase; and isolation of SFTSV from specimens.

Epidemiological investigation and data collection. Three SFTS clusters were identified in Zhejiang Province from 2018 to 2020, and there were four SFTS cases in each cluster. After confirmation of the index patient of each cluster, an epidemiological investigation was conducted as soon as possible. Data regarding the transmission route, epidemiological characteristics, and clinical characteristics were collected using a standardized questionnaire. The characteristics of 
three clusters were summarized and compared with those of other clusters.

Ethics statement. This experimental research was approved by the Ethics Committee of Zhejiang Provincial CDC. Human research was performed in accordance with the provisions of the Declaration of Helsinki. All participants were required to provide written informed consent to participate in the study.

\section{RESULTS}

Three SFTS clusters including 12 associated cases were identified in three counties of Zhejiang Province from 2018 to 2020 (Figure 1). Cluster A occurred in a village in Chun'an County, western Zhejiang Province. The first SFTS case was diagnosed on May 24, 2018; then, three secondary cases were confirmed. Cluster B occurred in a village in Shangyu County. The index patient experienced illness onset on June 12, 2018, was admitted to the local hospital on June 14, and was transferred to Shanghai East Hospital on June 21. The wife of the index patient experienced onset of illness on July 1, and SFTS was later confirmed on July 9 . The other two cases were identified later. Cluster $\mathrm{C}$ was reported in Keqiao County. The index patient first showed symptoms on May 9, 2020, was admitted to Shaoxing Municipal Hospital on May 11, and SFTS was confirmed on May 13. Three individuals who had close contact with the index patients had SFTS confirmed after that time.
TABLE 1

Demographic information of all SFTS cases

\begin{tabular}{llcll}
\hline Patient & Sex & Age, yr & Occupation & Relationship to the index patient \\
\hline Case A1 & Male & 64 & Farmer & Index patient \\
Case A2 & Male & 59 & Farmer & Son's father-in-law \\
Case A3 & Male & 61 & Farmer & Brother-in-law \\
Case A4 & Male & 65 & Farmer & Brother-in-law \\
Case B1 & Male & 70 & Farmer & Index patient \\
Case B2 & Female & 66 & Cleaner & Wife \\
Case B3 & Male & 21 & Worker & Grandson \\
Case B4 & Female & 47 & Cleaner & Daughter \\
Case C1 & Female & 80 & Farmer & Index patient \\
Case C2 & Female & 54 & Farmer & Daughter-in-law \\
Case C3 & Male & 72 & Farmer & Neighbor \\
Case C4 & Male & 49 & Farmer & Neighbor \\
\hline
\end{tabular}

There were three index patients (a 70-year-old man, a 64year-old man, and an 80-year-old woman) who performed daily agricultural activities. The secondary cases involved six men and three women with a median age of 59 years (range, 21-72 years) (Table 1). Of all SFTS cases, four were fatal (three index patients and one secondary case patient); the overall case fatality rate was $33 \%$. Of note, the fatality rate for index patients was $100 \%$ and the fatality rate of secondary case patients was $11 \%$. The total secondary attack rate of close contacts was $30 \%(9 / 30)$. The secondary attack rates of cluster A, cluster B, and cluster C were 38\% (3/8), $21 \%(3 / 14)$, and

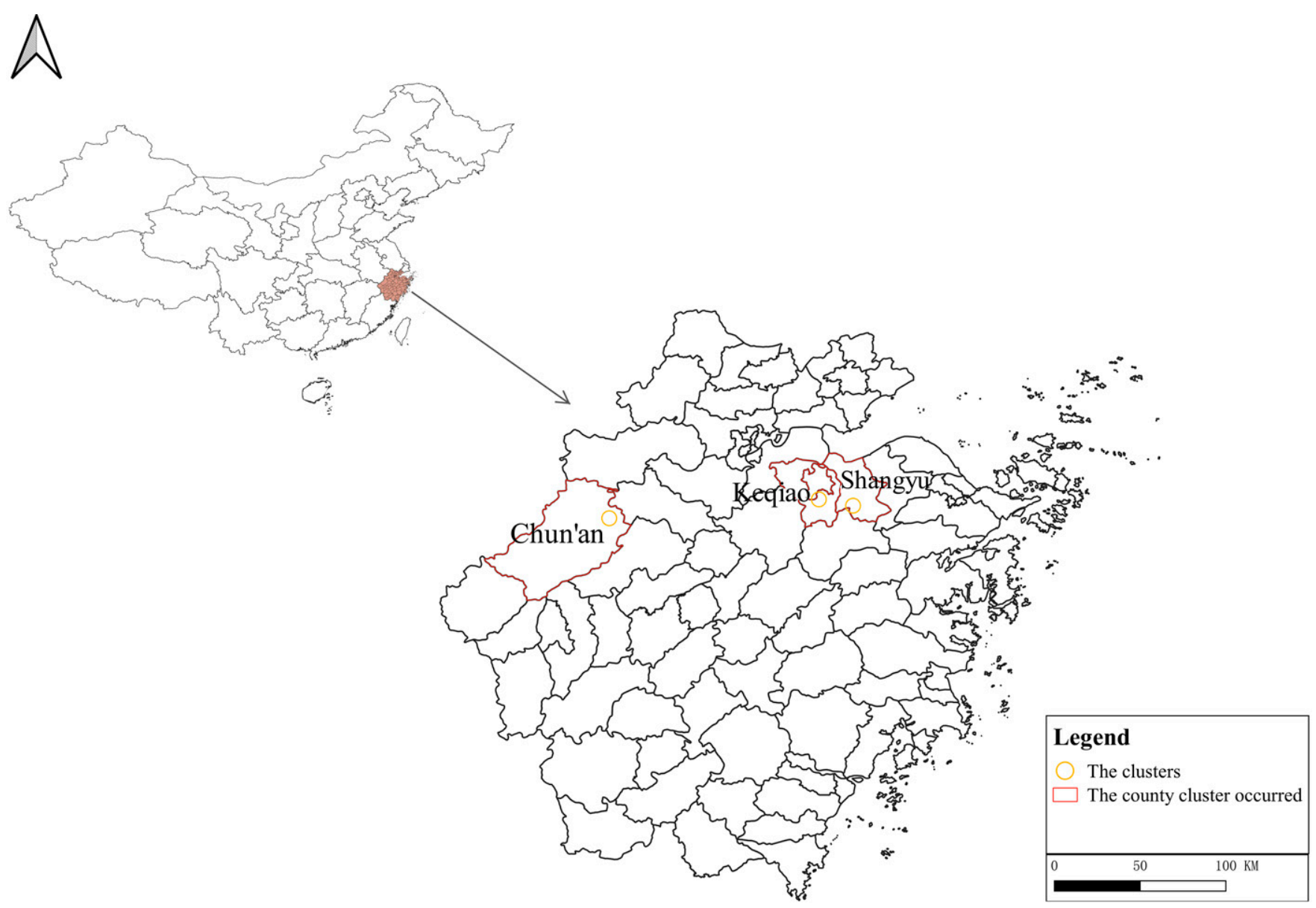

FIGURE 1. Geographical distribution of the three clusters. This figure appears in color at www.ajtmh.org. 
TABLE 2

Clinical information of all SFTS cases

\begin{tabular}{|c|c|c|c|c|c|c|c|c|c|c|c|c|}
\hline \multirow[b]{2}{*}{ Characteristic } & \multicolumn{4}{|c|}{ Cluster A } & \multicolumn{4}{|c|}{ Cluster B } & \multicolumn{4}{|c|}{ Cluster C } \\
\hline & 1 (Index) & 2 & 3 & 4 & 1 (Index) & 2 & 3 & 4 & 1 (Index) & 2 & 3 & 4 \\
\hline \multicolumn{13}{|l|}{ Clinical symptoms } \\
\hline Fever & Yes & Yes & Yes & Yes & Yes & Yes & Yes & Yes & No & Yes & Yes & No \\
\hline Fatigue & Yes & Yes & Yes & Yes & Yes & Yes & Yes & Yes & Yes & No & Yes & No \\
\hline Chills & Yes & Yes & Yes & Yes & No & No & Yes & Yes & No & Yes & Yes & No \\
\hline Diarrhea & Yes & No & Yes & No & Yes & No & Yes & Yes & Yes & No & No & No \\
\hline Headache & Yes & No & Yes & Yes & No & No & Yes & Yes & No & No & No & No \\
\hline Anorexia & No & No & Yes & Yes & No & No & No & No & No & No & No & Yes \\
\hline Myalgia & Yes & Yes & Yes & Yes & No & No & Yes & Yes & No & No & No & No \\
\hline Vomiting & No & Yes & No & No & No & No & Yes & Yes & Yes & No & No & No \\
\hline Gingival hemorrhage & No & No & No & No & No & No & No & No & No & Yes & No & No \\
\hline Conjunctival congestion & Yes & No & No & No & No & No & No & No & No & No & No & No \\
\hline \multicolumn{13}{|l|}{ Laboratory test } \\
\hline WBC & 2.13 & 1.78 & 0.94 & 3.63 & 1.83 & 1.14 & 3.50 & 0.90 & 2.34 & 4.60 & 2.40 & 1.53 \\
\hline PLT & 25.00 & 53.00 & 48.00 & 35.00 & 27.00 & 17.00 & 105.00 & 33.00 & 23.00 & 64.00 & 128.00 & 82.00 \\
\hline NEU & 1.30 & 1.30 & 0.90 & 3.30 & 1.26 & 0.90 & 2.44 & 0.50 & NK & NK & NK & NK \\
\hline LYM & 0.60 & 0.30 & 0.50 & 0.20 & 0.53 & 0.36 & 0.70 & 0.29 & NK & NK & NK & NK \\
\hline Elevated AST/ALT & ND & Yes & Yes & Yes & NK & Yes & Yes & Yes & Yes & NK & NK & NK \\
\hline RT-PCR & $P$ & $P$ & $\mathrm{P}$ & $P$ & ND & $\mathrm{P}$ & $P$ & $P$ & $\mathrm{P}$ & $\mathrm{P}$ & $\mathrm{P}$ & $\mathrm{P}$ \\
\hline
\end{tabular}

transcription polymerase chain reaction; WBC $=$ white blood cells.

$38 \%$ (3/8), respectively. Additionally, the interval from disease onset to confirmation was 4 days. The intervals from disease onset to confirmation of index patients and secondary case patients were 7 days and 4 days, respectively. All secondary case patients had a history of close contact with the index patients, including exposure to blood or body fluids.

Details of cluster A. The first case, case A1, was a 64-yearold man who worked as a farmer. He experienced chills, fever, systemic aches, fatigue, conjunctival congestion, anorexia, and diarrhea on May 20, 2018 (Table2). Case A1 was admitted to Chun'an County First People's Hospital on May 23. Routine blood tests indicated leukopenia and thrombocytopenia (white blood cell count, $2.13 \times 10^{9} / \mathrm{L}$; platelet count, 25.00 $\left.\times 10^{9} / \mathrm{L}\right)$. On May 24 , his specimen tested positive for SFTSV RNA by the Chun'an CDC, and he was transferred to Zhejiang Provincial People's Hospital. Of interest, one tick was found on his waist while he was in the hospital. On the evening of May 27, the patient experienced decreased blood pressure, heart rate, and oxygen saturation. Because his condition deteriorated, his family members asked the hospital to discharge him. Case A1 died on May 28, and his funeral was performed according to local customs. During the process of removing the intravenous infusion needle from the patient, a small amount of blood flowed out of the wounds, mouth, and nose of the corpse. The following five individuals had direct contact with the blood of the index patient: case A1's son's father-inlaw (case A2), brother-in-law (case A3), another brother-in-law (case A4), brother, and daughter-in-law's brother-in-law (Figure 2). None of these five individuals wore gloves or masks for personal protection when handling the corpse, and three individuals became ill. Moreover, another three individuals, including a driver and two neighbors, had a history of close contact with the index patient.

Case $\mathrm{A} 2$ reported the sudden onset of fever $\left(39.5^{\circ} \mathrm{C}\right)$, chills, fatigue, myalgia, and vomiting on June 5 , which was 8 days after exposure to the blood of the index patient (case A1). SFTS was confirmed on June 8. Case A3 and case A4 developed fever, fatigue, chills, headache, anorexia, and myalgia on June 6, and SFTS was confirmed on June 10.
Details of cluster B. The index patient, case B1, was a 70year-old man who performed daily cleaning of the river around the village. On June 12, 2018, he experienced fever, fatigue, and diarrhea. His symptoms were not mitigated after 2 days of infusion treatment at a local health center. He was transferred to Shangyu District Hospital on the June 17, and his white blood cell and platelet counts decreased $\left(1.16 \times 10^{9} / \mathrm{L}\right.$ and $70 \times 10^{9} / \mathrm{L}$, respectively) on the same day. On the evening of June 20 , the patient had sudden chest distress and shortness of breath, and his symptoms became more serious (Table 2). On June 21, he was transferred to Shanghai East Hospital. He was accompanied by his family members. On June 23 , his heart rate and blood pressure decreased. He experienced multiple organ failure and died. After he died, he was taken to his hometown in Shangyu, where he was placed in an ice coffin on June 24 and cremated on June 25 (Figure 3). A total of 11 individuals had direct contact with the patient while he was ill or with his corpse. Cases B2, B3, and B4 assisted in the removal of his endotracheal tube without the use of proper protection equipment and had contact with his blood, which had spattered out.

Case B2 was the wife of case B1. She was 66 years old and worked as a hospital cleaning staff member. She developed fever of unknown origin that reached a high of $40^{\circ} \mathrm{C}$ and had fatigue on July 1. She was hospitalized at Shangyu People's Hospital on July 3 and transferred to Shanghai East Hospital on July 6. On July 6, SFTS was confirmed. She died on July 9. Case B3, the grandson of case B1, experienced fever, fatigue, chills, headache, diarrhea, myalgia, and vomiting beginning on July 5 . SFTS was confirmed on July 8 . Case B4, the daughter of case B1, experienced fever, fatigue, chills, headache, diarrhea, myalgia, and vomiting on July 5 . On July 7 , she went to Shanghai Huashan Hospital. Her routine blood test showed a white blood cell count of $1.31 \times 10^{9} / \mathrm{L}$ and platelet count of $65 \times 10^{9} / \mathrm{L}$. SFTS was confirmed on July 10 .

Details of cluster $\mathbf{C}$. The index case was an 80-year-old woman with a history of paroxysmal atrial fibrillation. On May 9, 2020, she developed vomiting, diarrhea, and fatigue; however, fever did not develop. She was admitted to the Second 

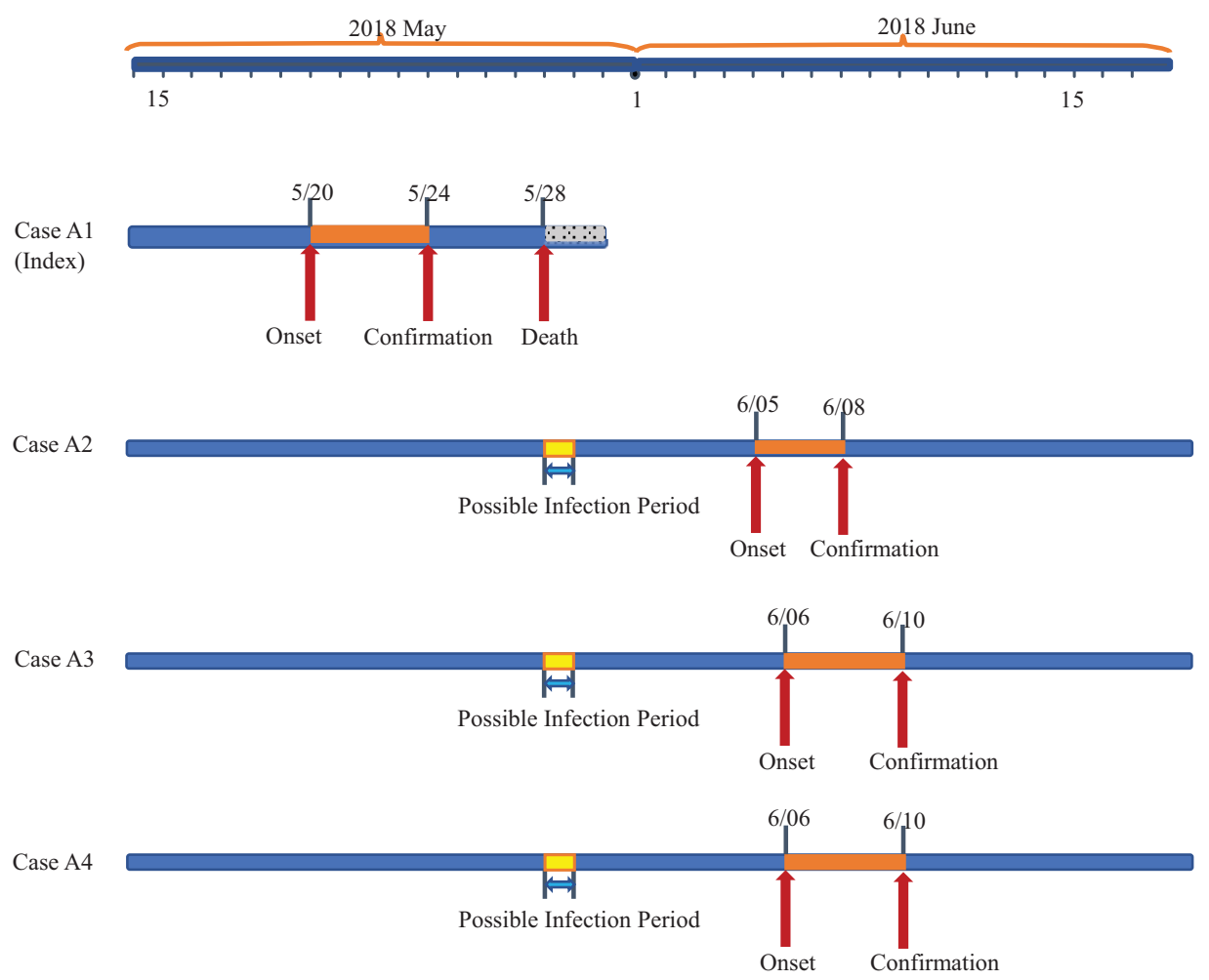

FIGURE 2. Timelines of key events for the three patients with severe fever with thrombocytopenia syndrome virus (SFTSV) infections in cluster A in 2018. This figure appears in color at www.ajtmh.org.

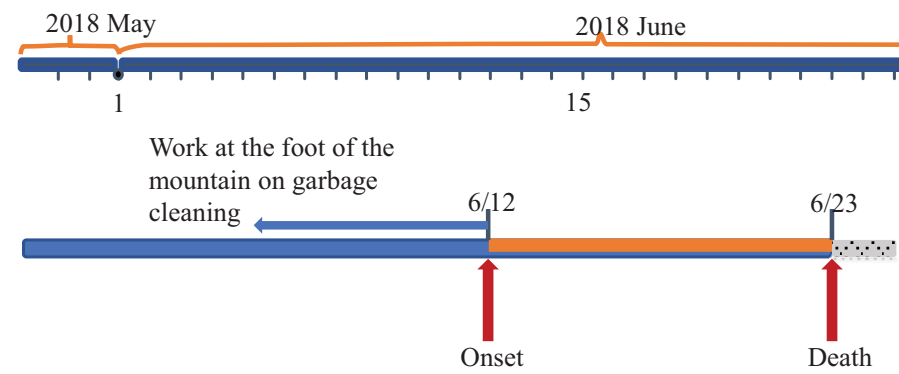

Case B2

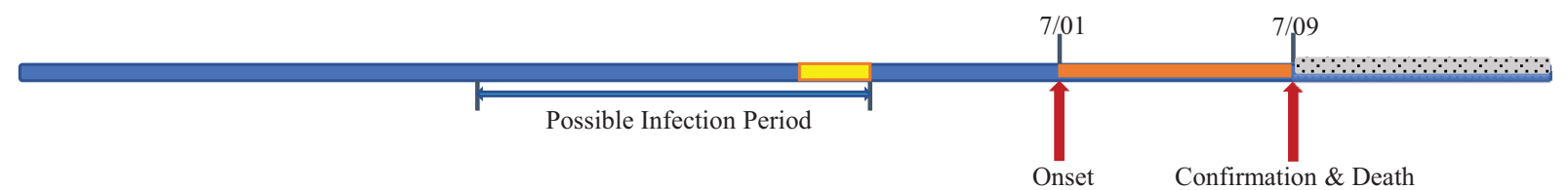

Case B3

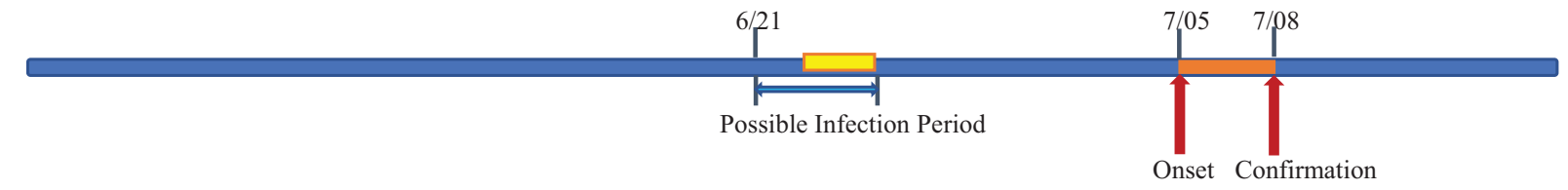

Case B4

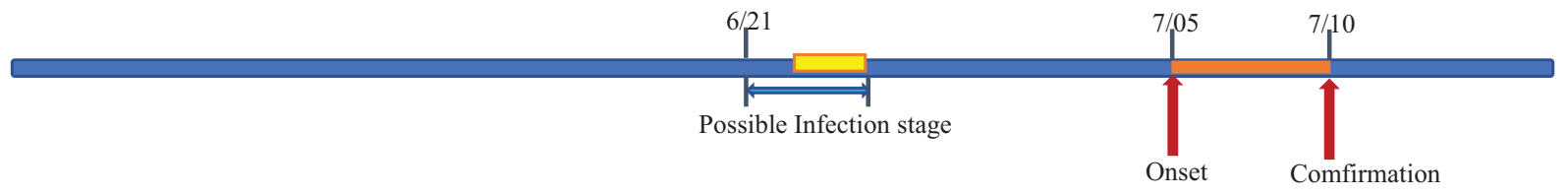

FIGURE 3. Timelines of key events for the three patients with severe fever with thrombocytopenia syndrome virus (SFTSV) infections in cluster B in 2018. This figure appears in color at www.ajtmh.org. 

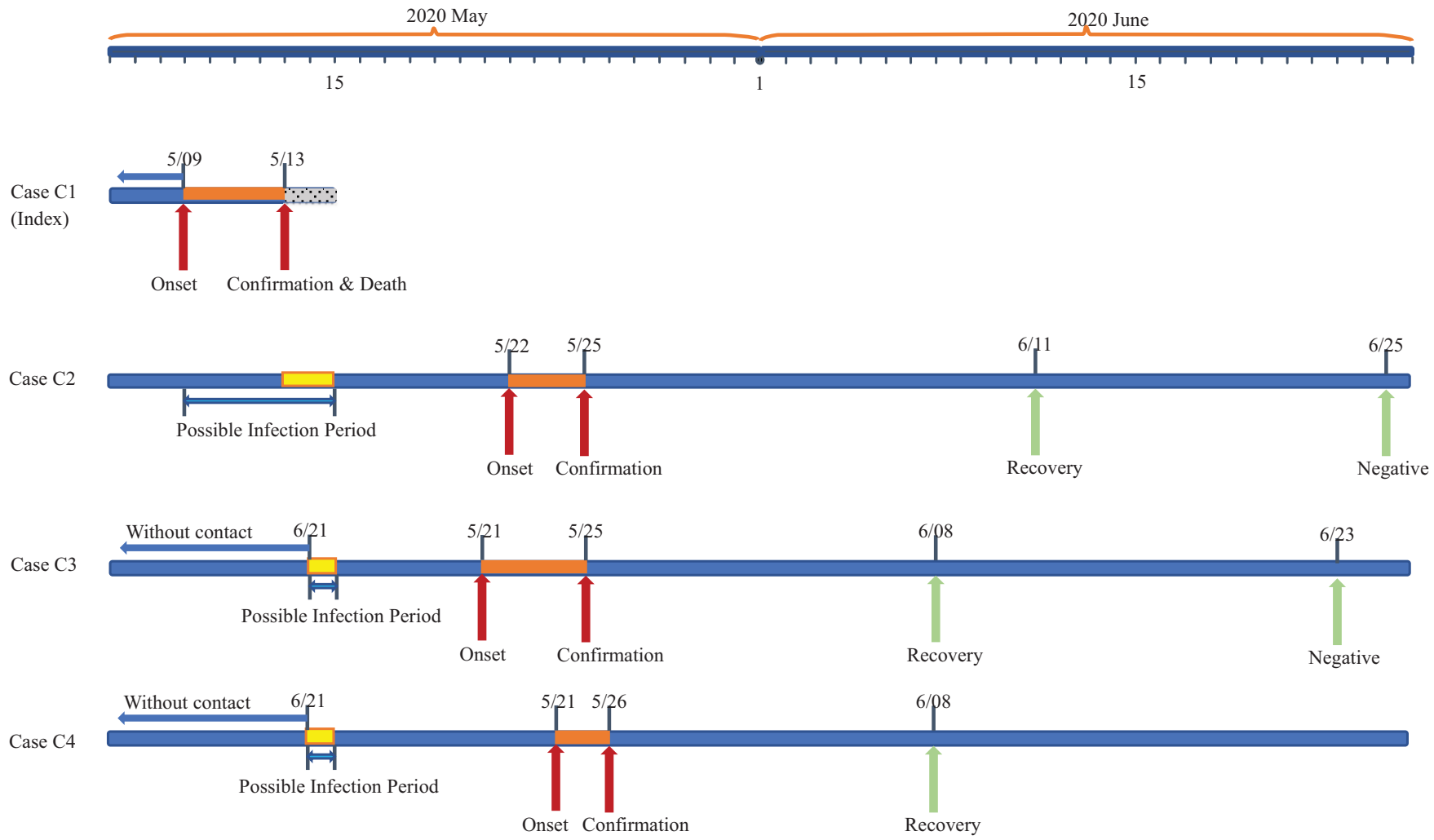

FIGURE 4. Timelines of key events for the three patients with severe fever with thrombocytopenia syndrome virus (SFTSV) infections in cluster C in 2020. This figure appears in color at www.ajtmh.org.

Hospital of Shaoxing on the same day (Table 2). On May 11, she returned to the local health center because of abdominal pain. She was transferred to Shaoxing Second Hospital on the same day. On May 12, she was considered to be infected with the SFTSV, and her blood sample was sent to the Keqiao CDC. On May 13, SFTS was confirmed. She was transferred from Shaoxing Second Hospital to the Municipal People's Hospital for further treatment. At 8:00 PM, the family members of the patient asked the hospital to discharge her, and she died at home at 10:00 PM (Figure 4).

Case $\mathrm{C} 2$ had cared for the index patients in the hospital. Cases C3 and C4 had contact with the wounds and blood of the corpse, helped carry the corpse to the funeral hall, and burned the clothes of the index patient. A total of eight individuals had direct contact with the index case or the corpse, and three persons were infected with the SFTSV.

Case C2, the daughter-in-law of case C1, was a 54-year-old woman who worked as a farmer. She developed fever $\left(39^{\circ} \mathrm{C}\right)$, chills, and gingival hemorrhage on May 22. SFTS was confirmed on May 25. Case C3, the neighbor of case C1, was a 72-year-old man with fever $\left(39.4^{\circ} \mathrm{C}\right)$, chill, fatigue, and occasional cough that developed on May 21. SFTS was confirmed on May 25. Case C4, the neighbor of case C1, was a 49-yearold man who first exhibited anorexia on May 24. SFTS was confirmed on May 26.

\section{DISCUSSION}

During this study, we investigated three SFTS case clusters during 2018 and 2020. Previous studies illustrated two SFTS case clusters that occurred in Zhejiang Province in
2014. ${ }^{22,25}$ Thereafter, the Ministry of Health of Zhejiang created a guideline indicating that every municipal CDC and the county CDC where more than five SFTS cases are confirmed must have the corresponding detection capacity for SFTSV. ${ }^{6}$ As a result, the interval from disease onset to confirmation began to decrease, no infections in healthcare workers occurred, and the scale and consequences of the incident became much smaller.

Among the three clusters, all index patients died of SFTS; one out of nine secondary case patients also died of SFTS. The fatality rate of secondary case patients was similar to that of sporadic case patients in Zhejiang Province. ${ }^{26} \mathrm{How}-$ ever, the fatality rate of index patients is as high as $100 \%$. Some factors may contribute to the significant high fatality rate. First, all index patients were elderly individuals (older than 64 years). Factors related to aging, such as decreased immune function and the presence of comorbidities with chronic diseases, can increase the risk of death. Second, the SFTS viral load of the index patients may have been higher than those of the secondary case patients. A high viral load may be associated with serious clinical symptoms. Third, the intervals from disease onset to confirmation of disease in the index patients were longer than those of the secondary case patients. One study reported that the OR of death increased with longer intervals from disease onset to disease confirmation. ${ }^{27}$ Nevertheless, the corpse of the SFTS patient is contagious; therefore, sufficient personal protective equipment should be used when dealing with the corpse.

Similar to the results of previous studies, all secondary cases had a history of contact with the blood and body fluids 
of the deceased. ${ }^{1,4,13}$ Cultural funeral practices in China have existed for thousands of years. Undoubtedly, the funeral is a meaningful farewell activity performed by humans for those who have lost their lives and involves profound cultural practices. ${ }^{28}$ Death and birth define human existence. The significance of birth is uncertain and is revealed through future events; however, the significance of death is proven by past activities. ${ }^{29}$ Therefore, the funeral comprises specific cultural activities that are rich and profound.

Since the establishment of the People's Republic of China, the traditional funeral rites have been simplified to only some symbolic procedures. ${ }^{30,31}$ However, before cremation, the specific funeral process includes cleansing the corpse, placing a shroud on the corpse, and preparing a funeral hall. Although Points for Prevention and Control of Spread of Severe Syndrome with Thrombocytopenia by Contact has clearly indicated that personal protection must be used when making contact with, disinfecting, and disposing of the dead, individuals' lack of medical knowledge, their respect for the dead, and their adherence to tradition have resulted in tragedy. ${ }^{32}$

SFTS is a high-risk and emerging infectious disease; therefore, the prevention of SFTS transmission from index patients to susceptible individuals is particularly important. To effectively reduce the incidence of SFTS, high-risk areas should be identified in advance and comprehensive tick prevention and control methods should be adopted in the most critical areas, including clearing breeding grounds, killing adult ticks, and providing health education. Patients with fatal cases should not be allowed to leave the hospital. After the patient dies in the hospital, the family members and medical workers should strictly follow the guidelines provided by Points for Prevention and Control of Spread of Severe Syndrome with Thrombocytopenia by Contact. ${ }^{32}$ Finally, it is necessary for local governments and health systems to establish longterm and effective training management and health promotion.

There were several limitations to this study. First, serum samples of close contacts were only tested for SFTSV RNA instead of lgM and lgG. Some contacts might be asymptomatic patients; therefore, the disease rate among close contacts may be underestimated. Second, the virus titer of SFTSV was not detected. No direct evidence confirmed that the viral load of index patients was higher than that of secondary case patients. Third, tick species, the density of tick species, and pathogens among tick species should be investigated in the future.

We summarized characteristics of three SFTS clusters in three counties of Zhejiang Province from 2018 to 2020. The index patients were older than the secondary case patients, and the intervals from disease onset to confirmation of the index patients were longer than those of secondary case patients. Notably, all index patients died and all secondary case patients were infected through contact with blood or bloody secretions of the corpse. These results indicated that more attention should be focused on fatal cases of SFTS. SFTS patients should not be discharged if they cannot be cured. When SFTS patients die, proper protective equipment should be worn by those in contact with the corpses. Corpses should be transferred directly from the hospital to the crematorium. The funeral should be performed with cremains to prevent the transmission of SFTSV. The results of this study should alert the local and state public health authorities to regulate the corpses of SFTS patients to prevent nosocomial transmission of SFTSV.

Received March 31, 2021. Accepted for publication May 7, 2021.

Published online July 12, 2021.

Acknowledgments: We thank the staff members at the hospitals, local health departments, and county-level, district-level, prefecture-level, and provincial-level CDCs for their valuable assistance with coordinating the data collection.

Financial support: This study was supported by National Natural Science Funds of China (no. 81872675), a grant from the Science Technology Department of Zhejiang Province (no. LGF20H260001), the state project for scientific \& technological development of the 13th 5-year plan in China (no. 2017ZX10303404008002), and the medical health science and technology project of Zhejiang Provincial Health Commission (no. 2019KY060).

Authors' addresses: Mingyong Tao, Ningbo University, Medical School, Ningbo, Zhejiang, China, E-mail: 1911101035@nbu.edu.cn. Ying Liu, Feng Ling, Rong Zhang, Xuguang Shi, Jiangping Ren, Song Guo, Jimin Sun, and Jianmin Jiang, Key Laboratory of Vaccine, Prevention and Control of Infectious Disease of Zhejiang Province, Zhejiang Provincial Center for Disease Control and Prevention, Hangzhou, China, E-mails: yliu@cdc.zj.cn, fengl@cdc.zj.cn, rzhang@ cdc.zj.cn, xgshi@cdc.zj.cn, jpren@cdc.zj.cn, sguo@cdc.zj.cn, jmsun@cdc.zj.cn, and jmjiang@cdc.zj.cn.

\section{REFERENCES}

1. Yu XJ, 2011. Fever with thrombocytopenia associated with a novel bunyavirus in China. N Engl J Med 364: 1523-1532.

2. Deng B, Zhou B, Zhang S, Zhu Y, Han L, Geng Y, Jin Z, Liu H, Wang D, Zhao Y, 2013. Clinical features and factors associated with severity and fatality among patients with severe fever with thrombocytopenia syndrome bunyavirus infection in northeast China. PLoS One 8: e80802.

3. Ding YP, Liang MF, Ye J, Liu Q, Xiong C, Long B, Lin W, Cui N, Zou $Z$, Song Y, 2014. Prognostic value of clinical and immunological markers in acute phase of SFTS virus infection. Clin Microbiol Infect 20: O870-0878.

4. Gai Z, Liang M, Zhang Y, Zhang S, Jin C, Wang SW, Sun L, Zhou N, Zhang Q, Sun Y, 2012. Person-to-person transmission of severe fever with thrombocytopenia syndrome bunyavirus through blood contact. Clin Infect Dis 54: 249-252.

5. Liu MM, Lei XY, Yu XJ, 2016. Meta-analysis of the clinical and laboratory parameters of SFTS patients in China. Virol $J$ 13: 1-6.

6. Sun J, Lu L, Wu H, Yang J, Liu K, Liu Q, 2018. Spatiotemporal patterns of severe fever with thrombocytopenia syndrome in China, 2011-2016. Ticks Tick Borne Dis 9: 927-933.

7. Kim KH et al., 2013.Severe fever with thrombocytopenia syndrome, South Korea, 2012. Emerg Infect Dis 19: 1892-1894.

8. Takahashi T et al., 2014. The first identification and retrospective study of severe fever with thrombocytopenia syndrome in Japan. J Infect Dis 209: 816-827.

9. Tran XC, Yun Y, Le Van An SHK, Thao NTP, Man PKC, Yoo JR, Heo ST, Cho N-H, Lee KH, 2019. Endemic severe fever with thrombocytopenia syndrome, Vietnam. Emerg Infect Dis 25: 1029.

10. McMullan LK et al., 2012. A new phlebovirus associated with severe febrile illness in Missouri. N Engl $J$ Med 367: 834-841.

11. Kohl C, Brinkmann A, Radonic A, Dabrowski PW, Nitsche A, Muhldorfer K, Wibbelt G, Kurth A, 2020. Zwiesel bat banyangvirus, a potentially zoonotic Huaiyangshan banyangvirus (formerly known as SFTS)-like banyangvirus in northern bats from Germany. Sci Rep 10: 1370.

12. Luo LM, Zhao L, Wen HL, Zhang ZT, Liu JW, Fang LZ, Xue ZF, Ma DQ, Zhang XS, Ding SJ, 2015. Haemaphysalis longicornis ticks as reservoir and vector of severe fever with thrombocytopenia syndrome virus in China. Emerg Infect Dis 21: 1770. 
13. Tang $X$ et al., 2013. Human-to-human transmission of severe fever with thrombocytopenia syndrome bunyavirus through contact with infectious blood. J Infect Dis 207: 736-739.

14. Liu JW et al., 2014. Prevalence of SFTSV among Asian house shrews and rodents, China, January-August 2013. Emerg Infect Dis 20: 2126-2128.

15. Niu G, Li J, Liang M, Jiang X, Jiang M, Yin H, Wang Z, Li C, Zhang $Q$, Jin C, 2013. Severe fever with thrombocytopenia syndrome virus among domesticated animals, China. Emerg Infect Dis 19: 756.

16. Huang XY, Du YH, Wang HF, You AG, Li Y, Su J, Nie YF, Ma HX, Xu BL, 2019. Prevalence of severe fever with thrombocytopenia syndrome virus in animals in Henan Province, China. Infect Dis Poverty 8: 56.

17. Kida K et al., 2019. A case of cat-to-human transmission of severe fever with thrombocytopenia syndrome virus. Jpn J Infect Dis 72: 356-358.

18. Liu Y, Li Q, Hu W, Wu J, Wang Y, Mei L, Walker DH, Ren J, Wang Y, Yu XJ, 2012. Person-to-person transmission of severe fever with thrombocytopenia syndrome virus. Vector Borne Zoonotic Dis 12: 156-160.

19. Chen H, Hu K, Zou J, Xiao J, 2013. A cluster of cases of human-tohuman transmission caused by severe fever with thrombocytopenia syndrome bunyavirus. Int $J$ Infect Dis 17: e206-e208.

20. Jiang XL et al., 2015. A cluster of person-to-person transmission cases caused by SFTS virus in Penglai, China. Clin Microbiol Infect 21: 274-279.

21. Wang S, Li J, Niu G, Wang X, Ding S, Jiang X, Li C, Zhang Q, Liang $\mathrm{M}, \mathrm{Bi} Z \mathrm{Z}, 2015$. SFTS virus in ticks in an endemic area of China. Am J Trop Med Hyg 92: 684-689.

22. Gong Z, Gu S, Zhang Y, Sun J, Wu X, Ling F, Shi W, Zhang P, Li D, Mao H, 2015. Probable aerosol transmission of severe fever with thrombocytopenia syndrome virus in southeastern China. Clin Microbiol Infect 21: 1115-1120.
23. Moon $\mathrm{J}$ et al., 2018. Aerosol transmission of severe fever with thrombocytopenia syndrome virus during resuscitation. Infect Control Hosp Epidemiol 40: 238-241.

24. Zhan J, Wang Q, Cheng J, Hu B, Li J, Zhan F, Song Y, Guo D, 2017. Current status of severe fever with thrombocytopenia syndrome in China. Virol Sin 32: 51-62.

25. Sun J, Tang Y, Ling F, Chang Y, Ye X, Shi W, Zhang L, Chen Z, Lin H, Qiu Z, 2015. Genetic susceptibility is one of the determinants for severe fever with thrombocytopenia syndrome virus infection and fatal outcome: an epidemiological investigation. PLoS One 10: e0132968.

26. Sun J et al., 2014. Epidemiological characteristics of severe fever with thrombocytopenia syndrome in Zhejiang Province, China. Int J Infect Dis 25: 180-185.

27. Sun $J$ et al., 2016. Factors associated with severe fever with thrombocytopenia syndrome infection and fatal outcome. Sci Rep 6: 33175

28. Lin G, 2013. From "dying without a burial place" to "resting in peace": research on the reform of Chinese funeral service system under the four-dimensional visual threshold. J Zhejiang Univ Humanit Soc Sci 3: 1-2.

29. Ming W, 2012. The immortal of trailbreaker: the image and character of Fangxiang in ancient Chinese funeral ritual. $J$ Tsinghua Univ Soc Sci 2: 116-125.

30. Kun Q, 2014. Sonic expressions of cosmological awareness: a comparative study of funeral rituals among Han Chinese living in the Yangzi River Valley. Yearb Tradit Music 46: 159-169.

31. Chan CLW, Chow AYM, Ho SMY, Tsui YKY, Tin AF, Koo BWK, Koo EWK, 2005. The experience of Chinese bereaved persons: a preliminary study of meaning making and continuing bonds. Death Stud 29: 923-947.

32. National Health Commission of the People's Republic of China, 2011. Points for Prevention and Control of Spread of Severe Syndrome with Thrombocytopenia by Contact. Available at: http://www.nhc.gov.cn/zwgk/wtwj/201304/447234f04fb54e38 b91a2b1b900aa27d.shtml. Accessed August 12, 2011. 